\title{
What do people know about the senses? Understanding perceptions of variation in sensory experience
}

\author{
Christine Cuskley*1 and Charalampos Saitis2 \\ 1Linguistics and Centre for Behaviour and Evolution, Newcastle University, Newcastle Upon \\ Tyne, UK \\ ${ }_{2}$ Centre for Digital Music, Queen Mary University London, London, UK \\ *Corresponding author: christine.cuskley@ncl.ac.uk
}

\begin{abstract}
Academic disciplines spanning cognitive science, art, and music have made strides in understanding how humans sense and experience the world. We now have a better scientific understanding of how human sensation and perception function both in the brain and in interaction than ever before. However, there is little research on how this high level scientific understanding is translated into knowledge for the public more widely. We present descriptive results from a simple survey and compare how public understanding and perception of sensory experience lines up with scientific understanding. Results show that even in a sample with fairly high educational attainment, many respondents were unaware of fairly common forms of sensory variation. In line with the welldocumented under representation of sign languages within linguistics, respondents tended to underestimate the number of sign languages in the world. We outline how our results represent gaps in public understanding of sensory variation, and argue that filling these gaps can form an important early intervention, acting as a basic foundation for improving acceptance, inclusivity, and accessibility for cognitively diverse populations.
\end{abstract}

\section{Introduction}

Our senses form our window into the world. Understanding human sensory experience, and how it supports uniquely human endeavours like music and language, is key to a better understanding of what it means to be human. An essential component of this is not only understanding the commonalities in sensory experience, but understanding how sensory experience varies: not everyone experiences the world in the same way. Despite this, there is little research on how this high level scientific understanding is translated into knowledge for the public more widely. The goal of this research is to learn more about how much of a gap there is between the general public's understanding of the senses, and what scientists know about sensation and perception.

Sensory experience exhibits considerable intra- and inter-individual variation. For example, synaesthesia - a phenomenon where stimulus in one sensory modality consistently elicits a response from two or more modalities (e.g., seeing colours when hearing a particular sound) is more common than previously understood, affecting close to $5 \%$ of the population (Simner \& Carmichael, 2015). There is also some systematic cultural variation: speakers of tone languages are significantly more likely to have perfect pitch (Deutsch et al., 2009), and while focal colours are broadly shared (Regier et al., 2005), color category boundaries vary considerably across cultures (Cook et al., 2005) and over time (Barley, 1974). Recent viral phenomena, in particular the gold and white/black and blue dress 
(Gegenfurtner et al., 2015; Rabin et al., 2016) and Yanny/Laurel audio (Pressnitzer et al., 2018) have revealed that awareness of sensory and perceptual variation is of inherent interest. However, we know little about what people already know about sensory variation, making efforts to create impactful public engagement difficult. While efforts in science communication about the senses are fairly common, they tend to focus primarily on the uniformity of human sensory experience (Fleur, 2017), and mainly take a brain-based perspective which tends to focus on vision and visual illusions (e.g., https://camera-obscura.co.uk).

\section{Assessing general knowledge of the senses}

We identify two major open questions regarding general knowledge of the senses. Fist, while various curricula (e.g., Key stage 2-3 primary curriculum in the UK) do cover human sensation and perception, the approach tends to focus on physical structures (e.g., how a particular part of the ear responds to vibration, or how waves of light enter the eye) rather than individual sensory or perceptual experience. Second, although most people have come across information about the senses in some early educational context, it's unclear how this translates into knowledge and understanding later on. Using a short, simple survey, we aim to assess what people know or understand about both variations in sensory experience and sensation and perception in general terms.

\section{Methods}

\section{Participants}

Ethics approval was obtained for the survey from Newcastle University, all participants were over 18 years of age. 148 participants completed the survey in total, which took approximately 5-10 minutes to fill out. 48 volunteered via social media or were sent the survey by a friend. To expand the sample particularly to include participants outside the researchers' social networks and extend the educational diversity of the sample, a further 100 participants were recruited via Mechanical Turk and paid $\$ 1.50$ for completing the short survey.

\section{Materials \& Procedure}

The survey included two sections, the first with 6 questions and the second with 7 questions. The full content of the survey is available in Appendix A, and was implemented using Jisc online surveys (onlinesurveys.ac.uk). Participants first consented to the terms of participating before continuing to the first section. Here, they were explicitly instructed to respond based on what they already know and specifically asked not to do even light research on the topics within the survey. We underscored that we were interested in intuitions and told participants that they would be given detailed information about the topics covered in the survey after they completed it.

The first section focused on information about the participant, including participants' age, where they live, where they grew up, their level of education, and any specialist subject areas. If possible, we aim to see whether responses to sensory related questions differ between different 
education levels and areas of study. Finally they were asked whether they identify as being either blind or visually impaired $1, \mathrm{~d} /$ Deaf or hard of hearing, having chronic mental health issues, or other chronic health issues. We anticipate that having lived sensory experience with differs markedly from the experience of most people (in the case of d/Deaf, hard of hearing, blind, or partially sighted individuals) may affect responses to the questions on sensory experience. Likewise, although we did not collect any specific detail on chronic mental or other health issues, it is possible that dealing with chronic mental or other health issues gives an individual a different perspective on the potential for variation in individual experience.

The second section focused on the participants' knowledge about sensory variation and sensation and participation. First, there was an open ended question asking participants 'How many senses do we have?'. Question 2 was a multiple choice question focusing on how the senses relate to each other, with the following options:

- They're separated and work independently in the brain.

- They work mostly independently in the brain, but sometimes interact.

- They are highly connected in the brain and work together, but can work independently.

- They are fundamentally connected and almost never work independently.

Question 3 focused on a handful of forms of sensory and perceptual variation: synaesthesia, ASMR, prosopagnosia, aphantasia, anosmia, misphonia, proprioception, amusia, CIPA, labyrinthitis, and color blindness. For an overview of each of these phenomena, see the detailed debrief (https://bit.ly/senses-debrief). For each of these phenomena, participants were asked to select one of the following options:

- I have never heard of this

- Seems familiar but I don't know what it is

- I have a vague idea of what it is

- I know what this is

- I have this

Question 4 focused on sensory loss. For vision, hearing, smell, taste, touch, and proprioception2, participants were asked whether the loss of the sense would have a very negative effect, a negative effect, cause mild discomfort, or be no different. Here, we aim to get a sense of how people without sensory loss may perceive the experience of those who do have some sensory loss. For example, significant stigma exists surrounding sight and hearing loss, despite the fact that a normal quality of life is possible with either if reasonable accessibility accommodations are in place. We aim

\footnotetext{
1 Note that within the survey the term 'Blind or visually impaired' was used. However, with the exception of accurately describing the methods as they were undertaken, we use the term 'partially sighted' throughout, we now understand to be the preferred alternative for 'visually impaired'. We recommend any adaptation of the survey for future work use this preferred term, and this is also noted in Appendix A.

2 Being aware that many people are not familiar with the concept of proprioception (indeed, our results confirmed this), we defined this as "ability to perceive one's own body in space" within the survey.
} 
to assess whether this stigma extends to other sensory modalities, and gain a better sense of how widespread it is.

The final three questions focused on cultural variation. Question 5 asked participants how many spoken languages they thought there were in the world, while question 6 asked how many signed languages they thought there were. Each of these was multiple choice with the options 1, 2-10, 10-50, 50-100, 100-500, 500-2000, 2000-5000, and 5000+. Here, we expect participants to under estimate the diversity of signed languages in particular, since up until the last few decades, there was a widespread misconception even within the scientific community that sign languages were merely a form of gesture, which even up until the latter half of the 20th century, was frequently assumed to be 'universal' (Mirzoeff, 1995; Woll et al., 2001).

Finally, participants were asked to rate "how much [they] think sensory experience varies across cultures" for vision, hearing, smell, taste, touch, proprioception, and ability to sense the passage of time. This question aims to assess people's perceptions of cultural universals and diversity. A rise in the study of language universals in the latter half of the $20_{\text {th }}$ century largely pushed the notion that language can affect cognition - also known as the Sapir-Whorf hypothesis or linguistic determinism - into the margins particularly in cognitive science (Casasanto, 2008). However, recent work in cognitive science has shown that linguistic variation does correspond with some cognitive or perceptual variation, particularly in terms of spatial metaphors for time (Casasanto, 2008), color boundaries (Cook et al., 2005), smell and taste (Majid et al., 2018; Majid \& Burenhult, 2014), and spatial cognition (Majid et al., 2004). Here, we aim to see whether people generally understand various forms of sensory experience to be "hard coded", or whether they consider them to be subject to cross-cultural variation.

At the end of the survey, participants were directed to a detailed debrief that gave them information about the things mentioned in the survey, including the sensory phenomena from question 3 , information about sensory loss, and information about language and cultural variation in sensory experience. The debrief can be found at https://bit.ly/senses-debrief.

\section{Results}

\section{Demographics of respondents}

The majority of respondents $(88.5 \%$; 131$)$ were between $25-54$ (25-34: 72; 35-4: 41;45-54: 18), a small number of 18-24 year old respondents (8) and respondents over 55 (9). Overall, most respondents were from the United States $(102 ; 68.9 \%)$, with most of these coming from the Mechanical Turk sample (92). Of the remaining respondents, $12.8 \%$ (19) were from the UK, $11.5 \%$ (17) were from elsewhere in Europe, and the remaining 6.8\% were from Argentina (1), Brazil (4) and India (5). While the countries where respondents grew up were slightly more diverse (including e.g., India, Mexico, Uganda, the Phillipines), respondents overwhelmingly grew up in the US (101) or UK 
(9). Given this heavy skew, we do not break down any results to the sensory experience questions in terms of location.

Most respondents had a Bachelor's degree (47.3\%; 70) or higher (Masters: $20.3 \%$; 30, PhD: $13.5 \%$; 20$)$, with the remaining respondents having some college $(9.5 \%, 14)$, high school or equivalent $(6.8 \% ; 10)$, or choosing not to respond $(2.6 \% ; 4)$. Most respondents favoured STEM subjects $(43.2 \%$; 64), although many were also inter-disciplinary $(26.3 \%$; 39). Arts/Humanities $(14.2 \% ; 21)$, social sciences $(10.2 \% ; 15)$, and human/cognitive sciences $(3.5 \% ; 5)$ made up most of the rest of the sample, with a few respondents choosing no particular area $(2.6 \% ; 4)$.

We also asked respondents if they were d/Deaf or hard of hearing, blind or visually impaired, and/or had chronic mental or other health issues. Four participants identified as d/Deaf or hard of hearing, 10 identified as blind or visually impaired, and 2 identified as both d/Deaf or hard of hearing and blind or visually impaired. Almost $24 \%$ of respondents (35 in total) reported having a chronic mental health issue, while about $16 \%$ (24) of respondents reported some other chronic health issue 3.

\section{Perceptions of the senses}

Over $80 \%$ of respondents identified there as being 5 or 6 senses $(5: 64.9 \%, 92 ; 6: 18.2 \%, 27)$, with 13 respondents indicating $<4$ and 10 indicating 7 or more. This indicates some variation at the upper and lower ranges, with one respondent saying there were 22 senses. The responses on the lower and higher end were predominantly from Mechanical Turk (only one volunteer responded with neither 5 or 6), however, Mechanical Turk respondents also formed 2/3 of the sample.

While this may in part reflect the "traditional" teaching that there are 5 distinct sensory systems, interestingly, most people understood that the senses were connected, with only $8.7 \%$ (13) of

How do the senses relate to each other, if at all?

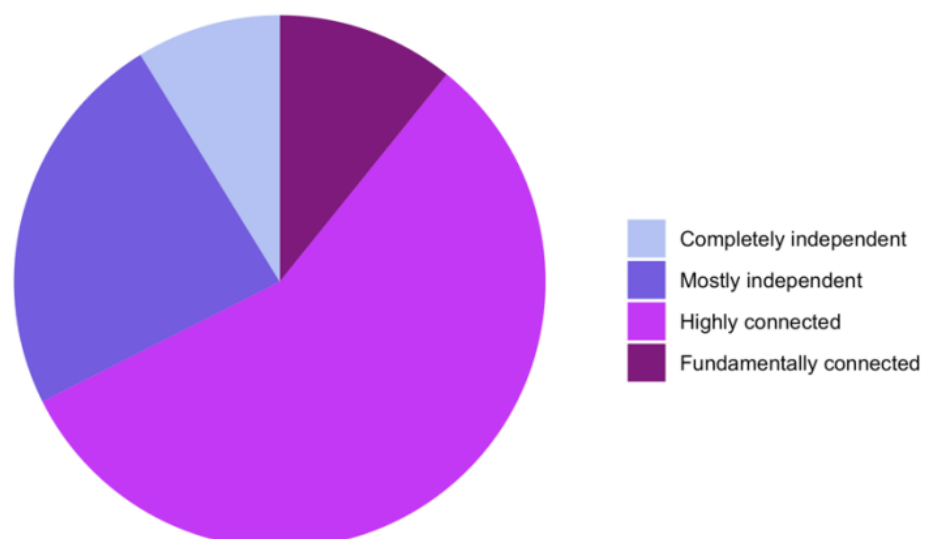

Figure 1 Answers to the question "How do the senses relate to each other, if at all?"

\footnotetext{
3 Although there was some overlap between these groups, given the potential sensitivity and stigma of mental health issues in particular (Casados, 2017) and the relatively small number of respondents identifying as $\mathrm{d} /$ Deaf/hard of hearing/blind/visually impaired, we will not give details about this overlap in order to avoid potentially singling out specific participants. Moreover, the goals of the survey were not designed to assess mental health issues in particular sub-populations, and thus, aside from the ethical considerations already mentioned, the representation of these populations is too low to draw any meaningful conclusions.
} 
participants overall indicating that they thought the senses worked completely independently, and most participants indicating that the senses were "highly connected in the brain and work together, but can work independently" (Figure 1).

Next participants were asked about their knowledge of (or experience with) several different kinds of sensory phenomena, most of which are not universal but have prevalence estimates ranging from extremely rare $(<0.001 \%$ for CIPA) to fairly common (30-40\% for ASMR; (Poerio et al., 2018)). One phenomena, proprioception, is simply the technical term for our "sixth sense" of our bodies' position in space, and is present in almost everyone (major deficits are generally caused by brain injury). Some of the remaining phenomena are primarily congenital (synaesthesia, aphantasia, CIPA, color blindness, amusia), others are usually acquired (labyrinthits, anosmia), but many may be either (anosmia, aphantasia, prosopagnosia). The remaining phenomena, misphonia and ASMR, less well understood in terms of either prevalence or emergence.

Figure 2 shows responses in terms of each phenomena, which is sized roughly relative to its prevalence in the general population, with proprioception (being more or less universal) sized largest. Of the phenomena rated, there is wide awareness of colour blindness (on average present in $4.5 \%$ of the population; (Deeb, 2005), with $73.6 \%$ of respondents reporting they know what it is. Synaesthesia

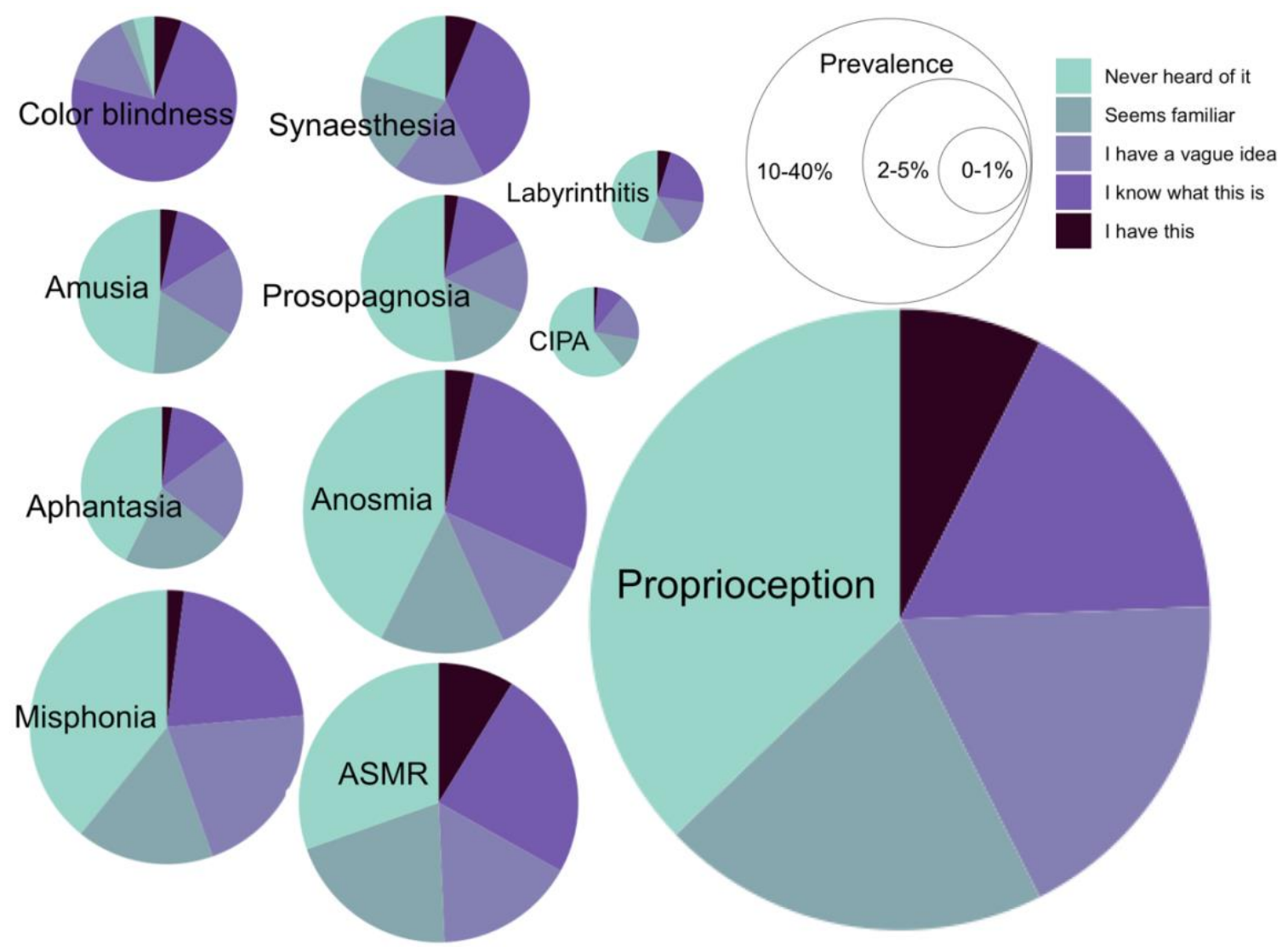

Figure 2 Awareness of various forms of sensory variation. 
has roughly the same prevalence in the population (approximately 4.4\% (Simner \& Carmichael, 2015), but more participants reported never having heard of it (20.3\%). Awareness of CIPA was lowest overall (60.8\% never heard of it), This is unsurprising given its extremely low prevalence and uneven spread (being strongly genetically linked, only 20 cases have been reported in the literature; (Reference, n.d.)). With the exception of of synaestheisa and color blindness, over $50 \%$ of participants reported not knowing what the phenomena were (either "never heard of it" or "seems familiar but I don't know what it is"), and this did not differ significantly based on education level. Most surprisingly, most people were unaware of proprioception, despite this often being thought of as a "sixth sense" in the study and scientific understanding of sensation and perception (Tuthill \& Azim, 2018).

The next question dealt with perceptions of sensory loss, and asked participants about the extent to which they think a loss of each sensory system would affect one's ability to lead a happy and fulfilling life. Here, we remove participants who reported having blindness and/or visual impairment or being $\mathrm{d} /$ Deaf or hard of hearing from the sample (a total of 16 participants). While their perspectives on this would be valuable, our sample does not have enough representation from either of these groups to draw any conclusions. We return to this issue in the discussion.

Overall, participants rated vision as being most likely to have a negative or very negative effect on a person's ability to lead a happy, fulfilling life ( $24.2 \%$ negative, $47.7 \%$ very negative). Hearing was rated similarly, though with a slightly higher proportion choosing a merely 'negative effect' ( $31.1 \%$ negative, $40.2 \%$ very negative). Touch and proprioception (the latter defined within the survey as "ability to perceive one's body in space") were rated similarly, with around two thirds of respondents reporting that this would be negative overall, but only just over a third rating it as "very negative" (Touch: $26.5 \%$ negative, $36.4 \%$ very negative; Proprioception: $26.5 \%$ negative, $39.4 \%$ very negative). Smell was rated as most likely to cause only mild discomfort (38.6\%) or no difference (16.7\%); likewise, just under $50 \%$ of participants rated taste loss as being likely to make either no 
difference (19.7\%) or cause only mild discomfort (26.5\%).

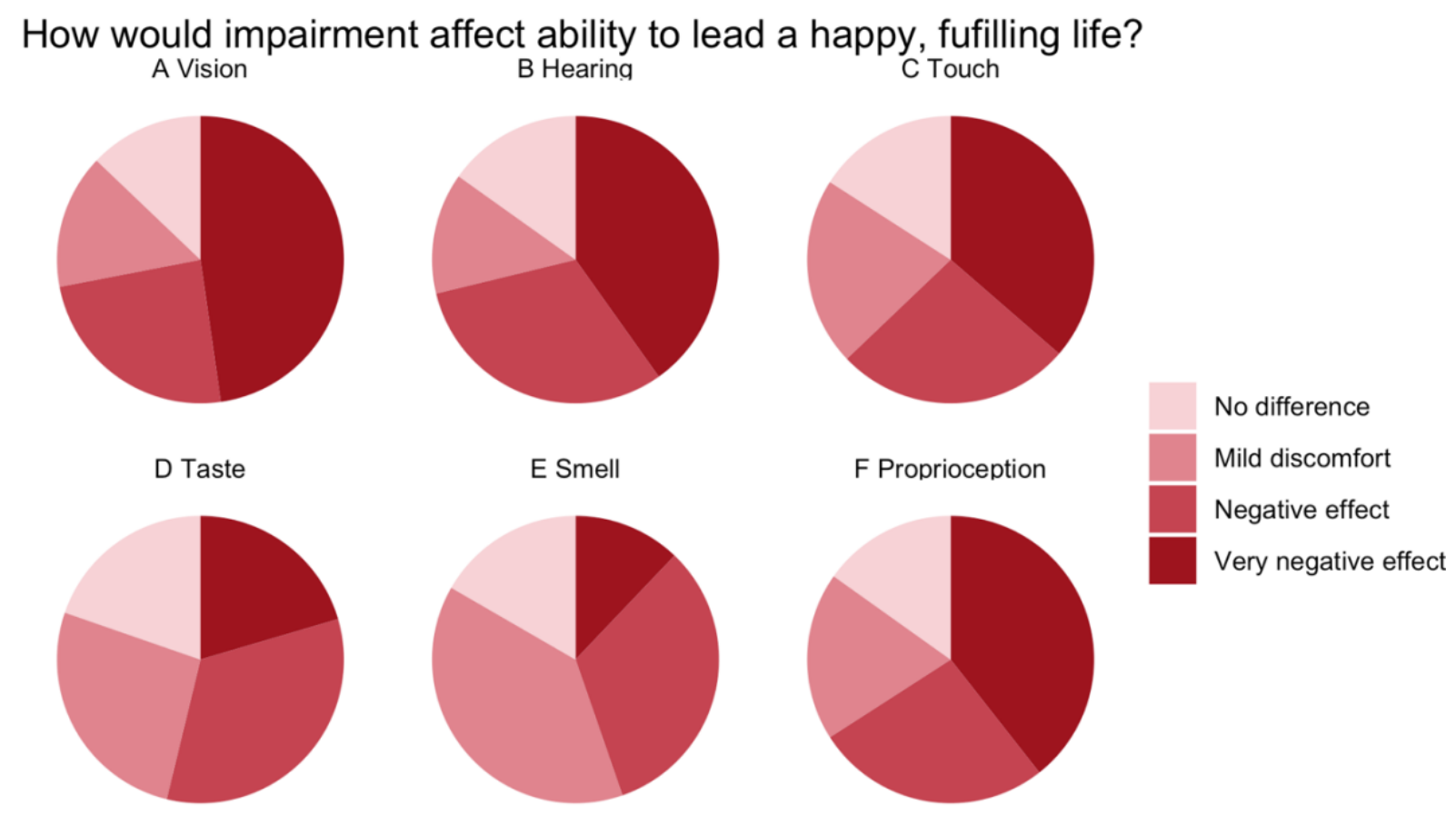

Figure 3 Perceptions of sensory loss. Participants were asked to rate how the loss of each sensory modality would affect one's ability to lead a happy, fulfilling life, and given the choices indicated in the legend.

In the questions about languages, many participants' understanding broadly lined up with scientific understanding. Most respondents correctly guessed that there were many thousands of spoken languages in the world (in different shades of green in Figure 4), with many correctly guessing that current estimates range over 5000 (Harmon, 1995). Estimates skewed lower for sign languages in general, but many respondents' guesses lined up with current estimates of there being a minimum of 1-200 sign languages and potentially hundreds more that are undocumented (highlighted in green in Figure 5; (Woll et al., 2001)). While some participants over estimated the likely number of sign languages in the world, most under estimated this number, with a small minority ( 8 participants) guessing there was only one sign language in the world. 
How many spoken languages are there in the world?

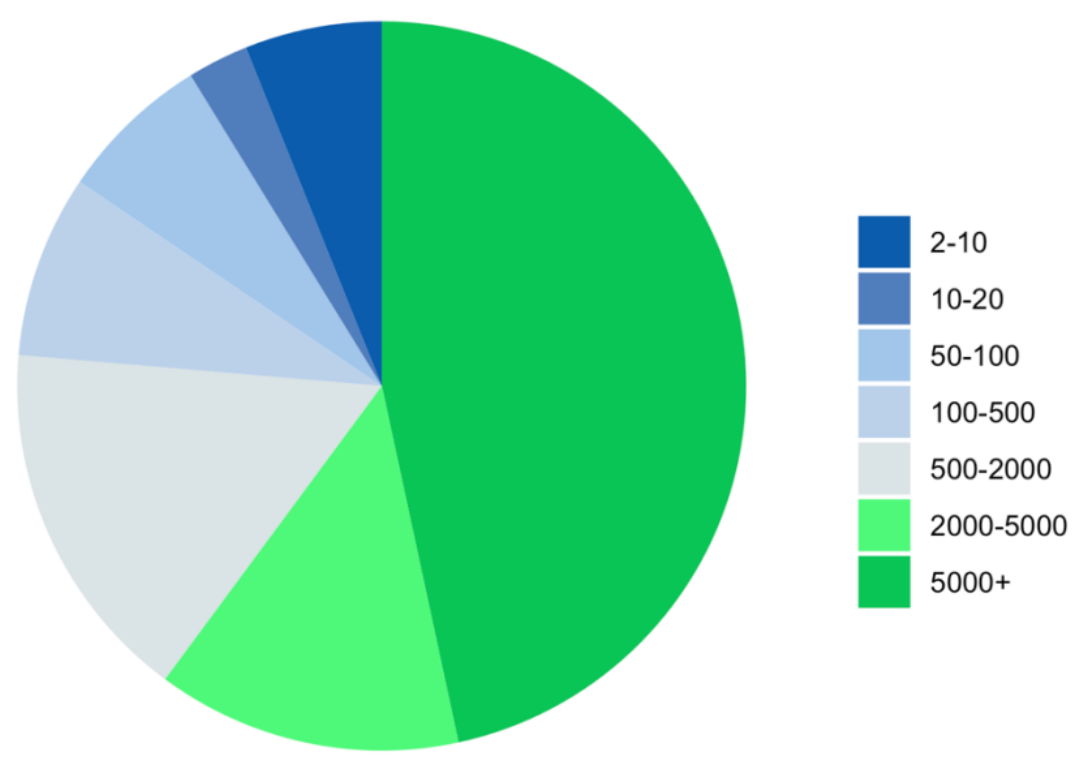

Figure 4 Respondents' estimates of the number of spoken languages in the world.

\section{How many signed languages are there in the world?}

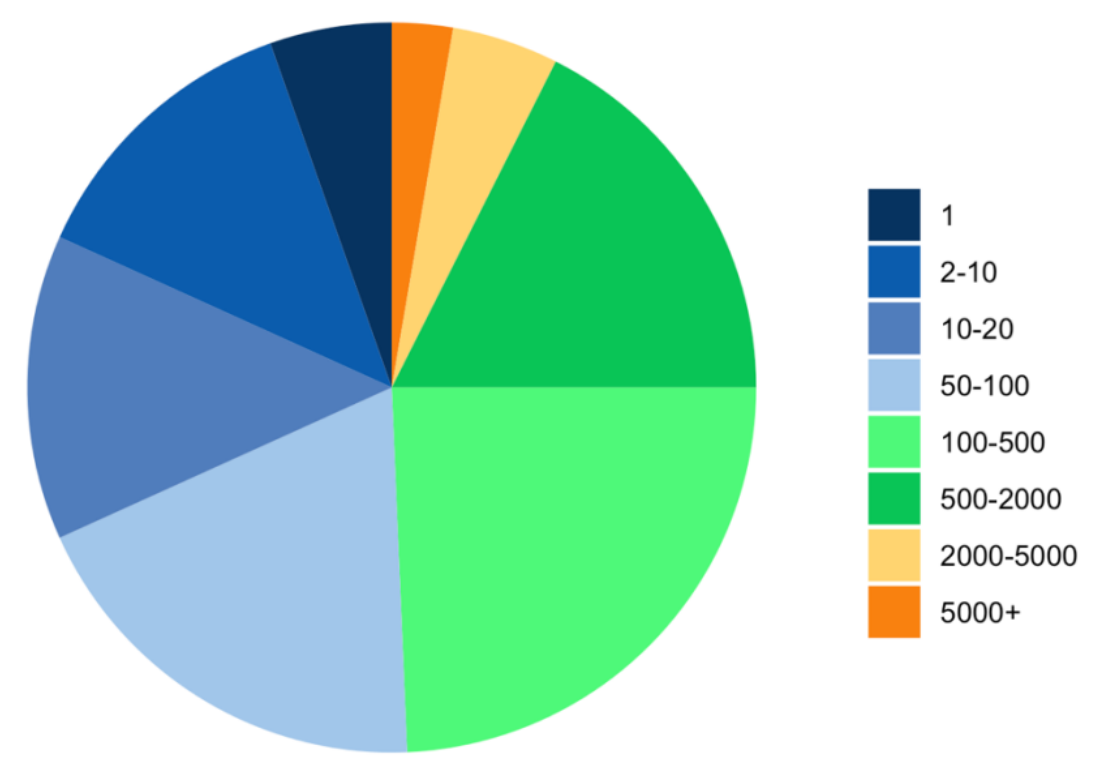

Figure 5 Respondents' estimates of the number of signed languages in the world.

Finally, we asked participants to think about sensory variation across cultures; results are shown in Figure 6. More than half of participants perceived vision, hearing, and proprioception (again, defined as "ability to perceive the body in space" within the survey) as varying not at all or 
almost never. In contrast, taste, smell, touch, and time were mostly rated as varying a little or a lot, with almost $75 \%$ of participants rating taste as exhibiting cultural variation.

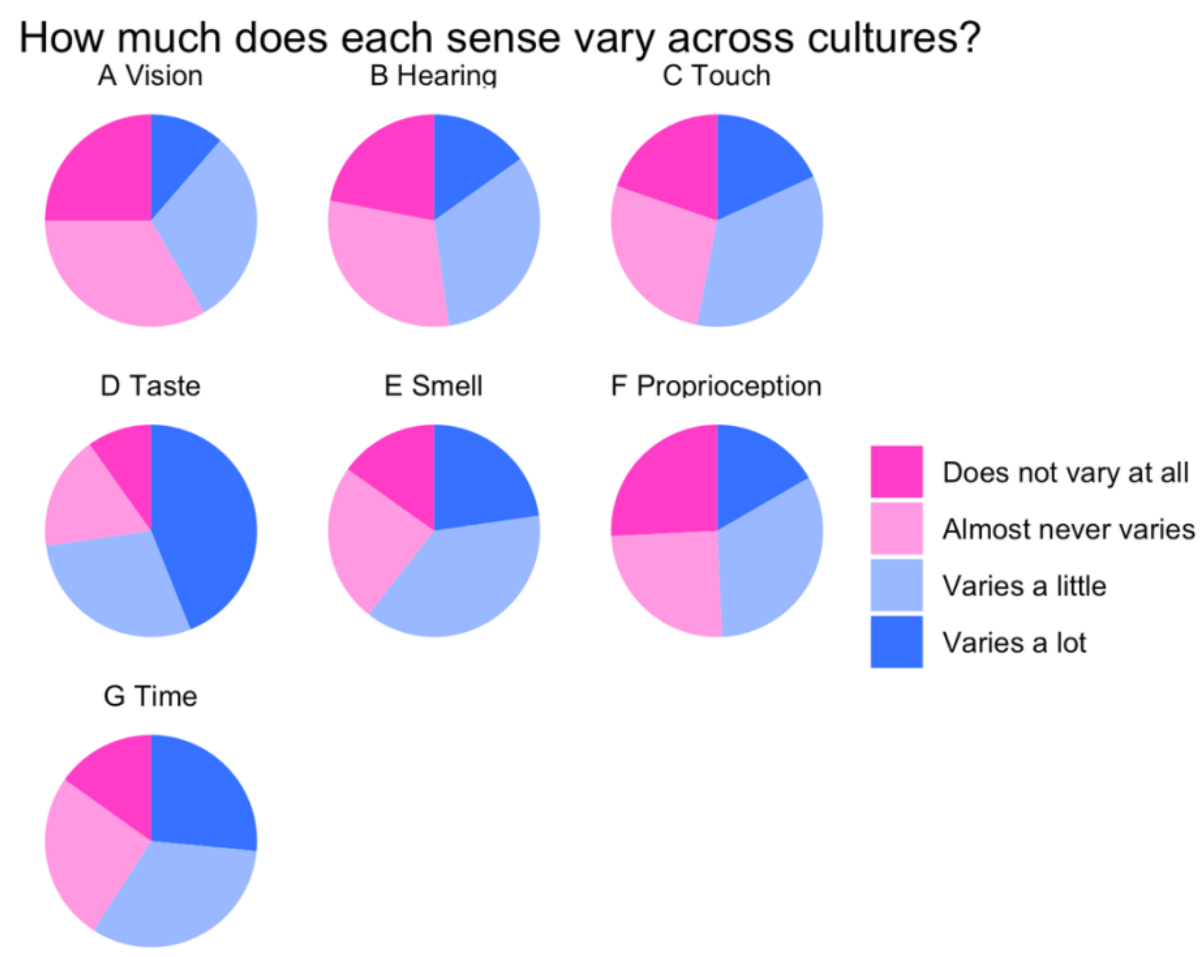

Figure 6 Perceptions of variation in sensory experience across cultures.

\section{Discussion}

This survey presented the first examination of what people know about the senses. Overall, while the " 5 senses" model of sensation and perception is widely recognised, people were mostly aware that scientists now consider the senses to be interconnected (Figure 1). This may be connected in part to surprisingly widespread awareness of synaesthesia - which is by definition an interconnectedness of sensory modalities within individual experience. Aside from colour blindness, synaesthesia was the only form of sensory variation we surveyed with more than half of respondents indicating awareness of it. In all the forms of sensory variation we surveyed, there did not seem to be any particular relationship between prevalence and awareness - participants were more aware of synaesthesia (which is relatively rare, affecting approximately $4.4 \%$ of the population) than proprioception (which is widespread).

Participants were also largely unaware of fairly widespread phenomena like misphonia and ASMR, though this may be because these phenomena are only beginning to be understood scientifically (Barratt \& Davis, 2015; Wu et al., 2014). This suggests a need for increased awareness surrounding these phenomena, particularly misphonia, which can be an unpleasant condition and contribute significantly to anxiety and even depression (Dozier et al., 2017; Wu et al., 2014). In some cases, our choice of terms within the survey may have contributed to rates of awareness. If we had used a more technical term for colour blindness like achromatopsia, awareness of this likely would 
have been lower. Likewise, people are likely to be aware of the loss of smell that can accompany a common cold, but are not aware of the term anosmia (or that this can have a longer-term neurological basis that may even be congenital). However, the importance of dedicated terms (and associated awareness) should not be under-estimated. For example, to return to misphonia, a person's knowledge that their extreme reaction to certain sounds is a genuine, named condition may come with some relief.

In terms of sensory loss, participants rated loss of vision, hearing, proprioception, and touch most negatively. Although participants were largely unaware of the very rare condition of CIPA, their responses in terms of touch indicate an intuitive understanding of how a loss of tactile sensation could be debilitating: in the case of CIPA, without the ability to feel pain, sufferers may experience life threatening injury or illness without realising it (Daneshjou et al., 2012). Participants rated loss of vision and hearing as likely to have an even more negative effect than touch loss. While loss in these modalities is ususally undesirable, loss in these modalities is in fact much less of a threat to quality of of life than loss of touch sensation. Participants' responses may reflect widespread stigma surrounding sight and hearing loss (Allen \& Birse, 1991; Erler \& Garstecki, 2002)

On the contrary, both d/Deaf /hard of hearing and blind/partially sighted populations have vibrant communities, and individuals with hearing loss are no more likely to be cognitively impaired than fully hearing individuals (Stokoe, 2001). These negative associations with certain types of sensory loss might have a kind of cyclical ratchet effect: the perception that sensory loss drastically lowers quality of life regardless could undermine accessibility and inclusion efforts, in turn making sensory loss more likely to cause isolation and a decrease in quality of life. For example, deaf or hard of hearing individuals who are denied access to sign language or signing communities are by definition language deprived (M. L. Hall et al., 2017; W. C. Hall, 2017), and so may be more likely to develop cognitive impairment as a result (W. C. Hall et al., 2017). This may in turn provide spurious support for the assumption that hearing loss itself negatively affects an individual's cognition or quality of life, when in fact this is caused by a lack of access to signing communities and resources. Further study should be done in particular on the negative perceptions of sight and hearing loss results could form the core of more effective accessibility and inclusion efforts. This could form an important component of building broad awareness that these differences in sensory experience are not inherently negative, but that inequitable access and inclusion can cause them to have negative effects.

Some of these attitudes were also reflected in the language related questions. While most participants correctly guessed the range of spoken languages on the planet, they were more likely to underestimate the number of sign languages. Interestingly, while taste and smell loss were rated as being least likely to have an effect on quality of life, they were also areas which were rated as being most likely to vary cross culturally. Cross-cultural variation in taste and smell is understudied, but recent work suggests that this is considerable. Vision and hearing were rated as being least likely to vary across cultures. However, research has in fact shown considerable cross-cultural variation 
particularly in colour perception and categorisation, and there is also some evidence of this for hearing, particularly with respect to speech sounds.

\section{Conclusions}

While this survey forms an important first step in understanding what people know about sensation and perception, it is only a start, and is very limited. While we collected information about educational background and location, for example, our small sample was too skewed for us to look at these factors in a systematic way. Future work should aim to focus on how understanding of sensation and perception may vary more systematically, for example across educational levels, different areas of study (e.g., those studying the humanities vs STEM subjects), and different locations. For example, early educational curricula in some places may lead to better understanding and awareness in a particular population. This, in turn, could lead to more systematic study of how this understanding is effectively built.

Likewise, it would be especially valuable to gather more data on perceptions of sensory experience in populations who have atypical sensory experience, particularly blind/partially sighted and d/Deaf or hard of hearing individuals. In particular, it would be revealing to know how they perceive their own sensory loss in more detail, to assess whether and how their lived experience contributes to more positive or negative perceptions, or if their perceptions in fact differ little from hearing and sighted populations. Individuals with sight or hearing loss are also by no means monolithic: deafness, hearing loss, blindness, and sight loss capture an incredible amount of individual variation not just in daily experience, but in life history. While some individuals are born with sensory loss, others acquire it at varying life stages which will inevitably affect the extent to which it alters their daily life.

Despite these limitations, this work forms an important first step to understanding our metaperception of the senses. Understanding more about how we perceive the overlap between our experience and the experience of others has significant implications for our understanding of metacognition and theory of mind, and can potentially help to support efforts in equity and inclusion.

\section{References}

Allen, M., \& Birse, E. (1991). Stigma and blindness. Journal of Ophthalmic Nursing \& Technology, $10(4), 147-152$.

Barley, N. F. (1974). Old English colour classification: Where do matters stand? Anglo-Saxon England, 3, 15-28. https://doi.org/10.1017/S0263675100000557

Barratt, E. L., \& Davis, N. J. (2015). Autonomous Sensory Meridian Response (ASMR): A flow-like mental state. PeerJ, 3, e851. https://doi.org/10.7717/peerj.851

Casados, A. T. (2017). Reducing the Stigma of Mental Illness: Current Approaches and Future

Directions. Clinical Psychology: Science and Practice, 24(3), 306-323.

https://doi.org/10.1111/cpsp.12206 
Casasanto, D. (2008). Who's Afraid of the Big Bad Whorf? Crosslinguistic Differences in Temporal Language and Thought. Language Learning, 58(s1), 63-79. https://doi.org/10.1111/j.14679922.2008.00462.x

Cook, R. S., Kay, P., \& Regier, T. (2005). Chapter 9-THE WORLD COLOR SURVEY DATABASE. In H. Cohen \& C. Lefebvre (Eds.), Handbook of Categorization in Cognitive Science (pp. 223-241). Elsevier Science Ltd. https://doi.org/10.1016/B978-0080446127/50064-0

Daneshjou, K., Jafarieh, H., \& Raaeskarami, S.-R. (2012). Congenital Insensitivity to Pain and Anhydrosis (CIPA) Syndrome; A Report of 4 Cases. Iranian Journal of Pediatrics, 22(3), $412-416$.

Deeb, S. S. (2005). The molecular basis of variation in human color vision. Clinical Genetics, 67(5), 369-377. https://doi.org/10.1111/j.1399-0004.2004.00343.x

Deutsch, D., Dooley, K., Henthorn, T., \& Head, B. (2009). Absolute pitch among students in an American music conservatory: Association with tone language fluency. The Journal of the Acoustical Society of America, 125(4), 2398-2403. https://doi.org/10.1121/1.3081389

Dozier, T. H., Lopez, M., \& Pearson, C. (2017). Proposed Diagnostic Criteria for Misophonia: A Multisensory Conditioned Aversive Reflex Disorder. Frontiers in Psychology, 8. https://doi.org/10.3389/fpsyg.2017.01975

Erler, S. F., \& Garstecki, D. C. (2002). Hearing Loss- and Hearing Aid-Related Stigma. American Journal of Audiology, 11(2), 83-91. https://doi.org/10.1044/1059-0889(2002/020)

Fleur, N. S. (2017, November 23). This Exhibition Will Help You Make Sense of Your Senses. The New York Times. https://www.nytimes.com/2017/11/23/arts/design/our-senses-exhibitionamerican-museum-of-natural-history.html

Gegenfurtner, K. R., Bloj, M., \& Toscani, M. (2015). The many colours of 'the dress'. Current Biology, 25(13), R543-R544. https://doi.org/10.1016/j.cub.2015.04.043

Hall, M. L., Eigsti, I.-M., Bortfeld, H., \& Lillo-Martin, D. (2017). Auditory Deprivation Does Not Impair Executive Function, But Language Deprivation Might: Evidence From a ParentReport Measure in Deaf Native Signing Children. The Journal of Deaf Studies and Deaf Education, 22(1), 9-21. https://doi.org/10.1093/deafed/enw054

Hall, W. C. (2017). What You Don't Know Can Hurt You: The Risk of Language Deprivation by Impairing Sign Language Development in Deaf Children. Maternal and Child Health Journal, 21(5), 961-965. https://doi.org/10.1007/s10995-017-2287-y

Hall, W. C., Levin, L. L., \& Anderson, M. L. (2017). Language deprivation syndrome: A possible neurodevelopmental disorder with sociocultural origins. Social Psychiatry and Psychiatric Epidemiology, 52(6), 761-776. https://doi.org/10.1007/s00127-017-1351-7

Harmon, D. (1995). The Status of the World's Languages as Reported in 'Ethnologue.' Southwest Journal of Linguistics, 14, 1-28.

Majid, A., Bowerman, M., Kita, S., Haun, D. B. M., \& Levinson, S. C. (2004). Can language restructure cognition? The case for space. Trends in Cognitive Sciences, 8(3), 108-114. https://doi.org/10.1016/j.tics.2004.01.003

Majid, A., \& Burenhult, N. (2014). Odors are expressible in language, as long as you speak the right language. Cognition, 130(2), 266-270. https://doi.org/10.1016/j.cognition.2013.11.004

Majid, A., Roberts, S. G., Cilissen, L., Emmorey, K., Nicodemus, B., O'Grady, L., Woll, B., LeLan, B., Sousa, H. de, Cansler, B. L., Shayan, S., Vos, C. de, Senft, G., Enfield, N. J., Razak, R. A., Fedden, S., Tufvesson, S., Dingemanse, M., Ozturk, O., ... Levinson, S. C. (2018). Differential coding of perception in the world's languages. Proceedings of the National Academy of Sciences, 115(45), 11369-11376. https://doi.org/10.1073/pnas.1720419115

Mirzoeff, N. (1995). Silent Poetry: Deafness, Sign, and Visual Culture in Modern France. Princeton University Press.

Poerio, G. L., Blakey, E., Hostler, T. J., \& Veltri, T. (2018). More than a feeling: Autonomous sensory meridian response (ASMR) is characterized by reliable changes in affect and physiology. PLOS ONE, 13(6), e0196645. https://doi.org/10.1371/journal.pone.0196645

Pressnitzer, D., Graves, J., Chambers, C., Gardelle, V. de, \& Egré, P. (2018). Auditory Perception: Laurel and Yanny Together at Last. Current Biology, 28(13), R739-R741. https://doi.org/10.1016/j.cub.2018.06.002 
Rabin, J., Houser, B., Talbert, C., \& Patel, R. (2016). Blue-Black or White-Gold? Early Stage Processing and the Color of 'The Dress'. PLOS ONE, 11(8), e0161090. https://doi.org/10.1371/journal.pone.0161090

Reference, N. I. of H. (n.d.). Chronic Insensitivity to Pain with Anhidirosis. Genetics Home Reference. Retrieved 21 August 2020, from https://ghr.nlm.nih.gov/condition/congenitalinsensitivity-to-pain-with-anhidrosis

Regier, T., Kay, P., \& Cook, R. S. (2005). Focal colors are universal after all. Proceedings of the National Academy of Sciences, 102(23), 8386-8391. https://doi.org/10.1073/pnas.0503281102

Simner, J., \& Carmichael, D. A. (2015). Is synaesthesia a dominantly female trait? Cognitive Neuroscience, 6(2-3), 68-76. https://doi.org/10.1080/17588928.2015.1019441

Stokoe, W. C. (2001). Deafness, cognition, and language (M. D. Clark, M. Marschark, \& M. A. Karchmer, Eds.). Gallaudet University Press.

Tuthill, J. C., \& Azim, E. (2018). Proprioception. Current Biology, 28(5), R194-R203. https://doi.org/10.1016/j.cub.2018.01.064

Woll, B., Sutton-Spence, R., \& Elton, F. (2001). Multilingualism: The global approach to sign languages. In C. Lucas (Ed.), The Sociolinguistics of Sign Languages (pp. 8-32). Cambridge University Press. https://doi.org/10.1017/CBO9780511612824.004

Wu, M. S., Lewin, A. B., Murphy, T. K., \& Storch, E. A. (2014). Misophonia: Incidence, Phenomenology, and Clinical Correlates in an Undergraduate Student Sample. Journal of Clinical Psychology, 70(10), 994-1007. https://doi.org/10.1002/jclp.22098

\section{Appendix A: Full Survey}

\section{Start Page}

This is a short survey on the senses conducted by Christine Cuskley at Newcastle University. It should take about 5-10 minutes to complete.

First we will ask you for some general information about yourself. Then you'll answer some questions about your thoughts about sensory experience and perception.

We want to know what you think or know about these topics to get a better idea of how general knowledge about sensation and perception lines up with scientific knowledge in this area. As such, please do not navigate away from the survey to do any light research - we're interested in your personal impressions about these questions.

If you're interested in the scientific understanding of these topics, we'll direct you to some further information at the end of the survey. If you have any questions about your data or the research, contact christine.cuskley@ncl.ac.uk.

Clicking the "Agree" box below indicates that:

- You agree that your anonymised data may be kept permanently in the research archives at Newcastle University and may be used by qualified researchers for teaching and research purposes.

- You agree that your anonymised data may be made publicly available for general use, e.g., released as part of scholarly publication.

- You voluntarily agree to participate and can stop or withdraw at any time without penalty.

[Agree or Disagree option with 'Next' to following page; participants only advanced if they agreed to the consent terms]

\section{General Information}

In this section, you'll give a little bit of information about yourself. This information is general - it cannot be used to identify you personally. 
1. How old are you?

- $18-24$

- 25-34

- $35-44$

- $45-54$

- $55-64$

- $65+$

2. What country do you live in? [free text]

3. If you grew up somewhere other than where you live now, list the country here. [free text]

4. Tell us a bit about your experience in education (select the highest level that applies)

- Prefer not to say

- High School or equivalent

- Some College or University

- Bachelor's Degree

- Master's degree

- Doctorate or similar

5. Broadly, what is/was your favourite subject of study (or, if you have an advanced degree, your area of specialisation). Select all that apply.

- Arts/Humanities

- Life/Physical Sciences

- Mathematics

- Technology/Engineering

- Social Sciences

- Human/Cognitive Sciences

6. Do you identify as belonging to any of the groups listed below? Select all that apply. [Note: if this survey is adapted, we recommend the use of the preferred term 'partially sighted' in lieu of 'visually impaired']

- Blind or visually impaired

- $d /$ Deaf or hard of hearing

- Chronic mental health issue (e.g., anxiety, depression)

- Other chronic health issue

Perceptions of the senses

1. How many senses do we have? [free text]

2. How do the senses relate to each other, if at all?

- They're separated and work independently in the brain

- They work mostly independently in the brain, but sometimes interact

- They are highly connected in the brain and work together, but can work independently

- They are fundamentally connected and almost never work independently 
3. Below are some words related to sensory experience. Rate them on the scale beside them based on what you already know about them - we want your first impressions, so please don't look anything up!

\begin{tabular}{|l|l|l|l|l|l|}
\hline & $\begin{array}{l}\text { Never heard } \\
\text { of it }\end{array}$ & $\begin{array}{l}\text { Seems } \\
\text { familiar, but I } \\
\text { don't know } \\
\text { what it is }\end{array}$ & $\begin{array}{l}\text { I have a vague } \\
\text { idea of what it } \\
\text { is }\end{array}$ & $\begin{array}{l}\text { I know what it } \\
\text { is }\end{array}$ & I have this \\
\hline Synaesthesia & & & & & \\
\hline ASMR & & & & & \\
\hline Prosopagnosia & & & & & \\
\hline Aphantasia & & & & & \\
\hline Anosmia & & & & & \\
\hline Misphonia & & & & & \\
\hline Proprioception & & & & & \\
\hline Amusia & & & & & \\
\hline CIPA & & & & & \\
\hline Labyrinthitis & & & & & \\
\hline $\begin{array}{l}\text { Color } \\
\text { Blindness }\end{array}$ & & & & & \\
\hline
\end{tabular}

4. Some people are born with different sensory abilities, and others can be lost or decline as a result of injury or normal aging. Rate how you think the loss or absence of each sense would affect a person's ability to lead a happy, fulfilling life in general (assuming medical intervention could not mitigate the loss - e.g., a visual impairment that cannot be corrected using glasses or contacts).

\begin{tabular}{|l|l|l|l|l|}
\hline & $\begin{array}{l}\text { Very negative } \\
\text { effect }\end{array}$ & Negative effect & Mild discomfort & No difference \\
\hline Vision & & & & \\
\hline Hearing & & & & \\
\hline Smell & & & & \\
\hline Taste & & & & \\
\hline Touch & & & & \\
\hline $\begin{array}{l}\text { The ability to } \\
\text { perceive one's } \\
\text { own body in } \\
\text { space }\end{array}$ & & & & \\
\hline
\end{tabular}

5. How many spoken languages do you think there are in the world?

- $2-10$

- $10-50$

- $50-100$ 
- $100-500$

- $500-2000$

- $2000-5000$

- $5000+$

6. Not everyone in the world can hear, so not all language is spoken. How many signed languages do you think there are?

- 1

- $2-10$

- $10-50$

- $50-100$

- $100-500$

- $500-2000$

- $2000-5000$

- $5000+$

7. There are many different cultures in the world. How much do you think sensory experience varies across cultures?

\begin{tabular}{|l|l|l|l|l|}
\hline & Varies a lot & Varies a little & $\begin{array}{l}\text { Almost never } \\
\text { varies }\end{array}$ & $\begin{array}{l}\text { Does not vary at } \\
\text { all }\end{array}$ \\
\hline Touch & & & & \\
\hline Taste & & & & \\
\hline Vision & & & & \\
\hline Hearing & & & & \\
\hline Smell & & & & \\
\hline $\begin{array}{l}\text { The ability to } \\
\text { sense one's own } \\
\text { body in space }\end{array}$ & & & & \\
\hline $\begin{array}{l}\text { Ability to sense } \\
\text { the passage of } \\
\text { time }\end{array}$ & & & & \\
\hline
\end{tabular}


